Chirurg 2015 $\cdot 86: 213$

DOI 10.1007/s00104-014-2827-6

Online publiziert: 7. März 2015

(c) Springer-Verlag Berlin Heidelberg 2015

P.M. Vogt

Klinik für Plastische, Hand- und Wiederherstellungschirurgie, Medizinische Hochschule Hannover

\title{
Plastisch-rekonstruktive Chirurgie und Präfabrikation von Geweben im interdisziplinären Komplikationsmanagement
}

schen Verfahren der plastischen Weichgewebsdeckung erfolgen kann, erfordern die mitunter komplexen Einzelfälle individuell an den Patienten angepasste Lösungen. Hierfür hat sich als Erfolg versprechender Ansatz in den letzten Jahren die Präfabrikation von Geweben entwickelt. Im Gegensatz zu alloplastischen Prothesen und Epithesen wird in der Regel ausschließlich körpereigenes Gewebe und biokompatible Matrix verwendet. Der Vorteil dieses Therapieprinzips liegt in einer Minimierung oder sogar Eliminierung biologischer Abstoßungs- und Fremdkörperreaktionen und - je nach Indikation - auch der Hebedefektmorbidität von Transplantatentnahmeregionen. Hiermit erreicht der rekonstruktive Chirurg die größtmögliche Annäherung an das Prinzip „Gleiches mit Gleichem“ zu ersetzen.

Im Mittelpunkt dieser Ausgabe von Der Chirurg 3/2015 stehen das Spektrum und die Möglichkeiten des plastisch-chirurgischen Komplikationsmanagements sowie das Prinzip der Präfabrikation, mit dem zukünftig noch eine bessere Annäherung an einen optimierten biologischen Ersatz sichtbarer Regionen und Körperfunktionen erzielt werden kann. Dabei reicht das Spektrum von gezüchtetem Röhrenknochen über präfabrizierte Kieferanteile, Trachea und Nase bis hin zu mitwachsenden Herzklappen.

Ich danke allen Autorinnen und Autoren und dem Springer-Verlag mit Frau Dr. Jutta Schneider für die Unterstützung bei der Realisierung des Heftes zu diesem wichtigen chirurgischen Thema.

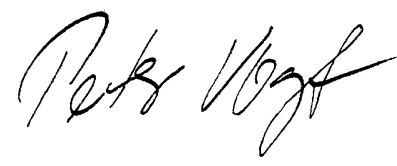

Univ.-Prof. Dr. Peter M. Vogt

\section{Korrespondenzadresse}

Prof. Dr. P.M. Vogt

Klinik für Plastische,

Hand- und Wiederherstellungschirurgie,

Medizinische Hochschule Hannover, Carl-Neuberg-Str. 1, 30625 Hannover

Vogt.Peter@MH-Hannover.de

Interessenkonflikt. P.M. Vogt gibt an, dass kein Interessenkonflikt besteht.
Wenngleich die Mehrzahl der anspruchsvollen Rekonstruktionen mit den klassi- 